\title{
Magnetization plateaus in dimerized spin-ladder arrays
}

\author{
D. C. Cabra \\ Departamento de Física, Universidad Nacional de La Plata, CC 67, 1900 La Plata, Argentina \\ and Facultad de Ingeniera, Universidad Nacional de Lomas de Zamora, Cno. de Cintura y Juan XXIII, \\ 1832 Lomas de Zamora, Argentina \\ M. D. Grynberg \\ Departamento de Física, Universidad Nacional de La Plata, CC 67, 1900 La Plata, Argentina
}

(Received 23 November 1999; revised manuscript received 24 February 2000)

\begin{abstract}
We investigate the ground-state magnetization plateaus appearing in spin- $\frac{1}{2}$ two-leg ladders built up from dimerized antiferromagnetic Heisenberg chains and dimerized zig-zag interchain couplings. Using both Abelian bosonization and Lanczos methods we find that the system yields rather unusual plateaus and exhibits massive and massless phases for specific choices or "tuning" of exchange interactions. The relevance of this behavior in the study of $\mathrm{NH}_{4} \mathrm{CuCl}_{3}$ is discussed.
\end{abstract}

\section{INTRODUCTION}

The study of low dimensional antiferromagnets is playing a major role and bringing new insights in our current understanding of collective spin fluctuations. ${ }^{1}$ Part of the fascination of this field is that in the extreme quantum limit of $S$ $=1 / 2$ there is a rather complex crossover between onedimensional (1D) Bethe ground states and 2D long-range order. By now it is well established that, at least within the strong-coupling regime, ladders with an even number of chains exhibit massive phases with purely short-range spin correlations. In contrast, odd-chain ladders display in general massless spin excitations resembling typical 1D ground states with power-law decaying correlations. A wealth of experimental investigations have confirmed these expectations for an increasing number of materials, such as families of low-dimensional cuprates like $\mathrm{Sr}-\mathrm{Cu}-\mathrm{O}$ and $\mathrm{La}-\mathrm{Cu}-\mathrm{O}$ compounds, ${ }^{1,2}$ which can be well described in terms of spin$1 / 2$ Heisenberg antiferromagnets confined to ladder geometries.

Surprisingly, recent measurements of magnetization curves in $\mathrm{NH}_{4} \mathrm{CuCl}_{3}$ crystals at high magnetic fields ${ }^{3}$ revealed rather unexpected features which contrast with the general behavior of spin excitations expected for these systems. $^{4-8}$ Specifically, this two-leg ladder $S=1 / 2$ dimerized compound displays two magnetization plateaus at onequarter and three-quarter of the saturation magnetization, irrespective of the external field direction. These results confront one of the central issues regarding the condition of fractional quantization for the appearance of massive spin excitations or magnetization plateaus under external varying fields. On general grounds this condition can be shown to $\mathrm{be}^{4-8}$

$$
p N S(1-\langle M\rangle) \in \mathcal{Z}
$$

where $p, N$, and $S$ stand, respectively, for the periodicity of the ground state, the number of coupled chains and the total spin per site, whereas $\langle M\rangle$ denotes the system magnetization normalized to its saturation value. Thus, at sufficiently low temperatures two coupled spin-1/2 dimerized chains $(p=N$ $=2$ ) should exhibit, if any, two plateaus at $\langle M\rangle=0$ and $1 / 2$. However, observations at temperatures down to $0.5 \mathrm{~K}$ and magnetic fields up to $35 \mathrm{~T}$ carried out $\mathrm{in}^{3}$ showed no evidence of these latter plateaus. The situation is rather intriguing since apart from $3 \mathrm{D}$ effects in $\mathrm{NH}_{4} \mathrm{CuCl}_{3}$ as well as low-temperature structural transitions which cannot be ruled out, the magnetization of a number of low dimensional halides and pnictides, e.g., $\mathrm{KCuCl}_{3},{ }^{3,9,10} \mathrm{Cs}_{2} \mathrm{CuCl}_{4},{ }^{11}$ seems to be in fair agreement with Eq. (1).

Motivated by these conflicting observations, recent theoretical studies ${ }^{12,13}$ pointed out that two-leg ladder antiferromagnetic systems can exhibit vanishing spin gaps depending on the manner in which the array of coupling exchanges is realized. The key issue is that the interplay between massive generating mechanisms such as dimerization and interchain couplings eventually yield no cost in energy to create spin excitations under magnetic fields. Therefore, the system switches from one ground state to another (short or long ranged), depending in a highly nontrivial way on the particular choice or "tuning" of coupling exchanges. This is an ubiquitous sign of the importance of quantum fluctuations of individual spins on the ground state. Depending on the exchange interactions, fluctuations can manifest themselves collectively into many possible ground states, particularly in lower dimensions where their effects are most pronounced.

In this work we further analyze these findings by means of two independent and complementary techniques such as Abelian bosonization ${ }^{4-8}$ and Lanczos diagonalization ${ }^{14}$ of finite systems. We consider ladders of richer topologies including both frustrated interactions and dimerization along the interchain and intrachain couplings. Specifically, we focus attention on two dimerized spin-1/2 chains interacting through an isotropic Hamiltonian of the form

$$
\begin{aligned}
H= & \sum_{a=1,2, n=1}^{L} J_{n}^{(a)} \vec{S}_{n}^{(a)} \cdot \vec{S}_{n+1}^{(a)}+J_{2} \sum_{n=1}^{L}\left[\left(1-\delta_{2}\right) \vec{S}_{n}^{(1)} \vec{S}_{n}^{(2)}\right. \\
& \left.+\left(1+\delta_{2}\right) \vec{S}_{n}^{(1)} \vec{S}_{n+1}^{(2)}\right]
\end{aligned}
$$




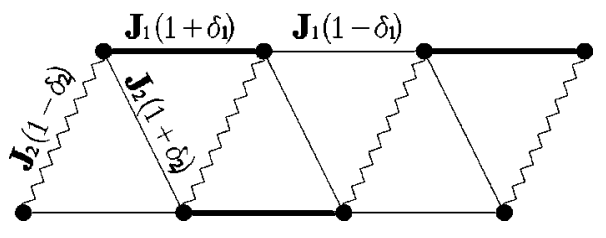

FIG. 1. Schematic view of doubly alternating zig-zag ladders showing both interchain $\left(J_{1}\right)$, and intrachain $\left(J_{2}\right)$ exchange couplings along with their respective dimerization parameters $\delta_{1}$ and $\delta_{2}$.

where the $\vec{S}_{n}$ denote spin-1/2 operators associated to site $n$. The array of coupling exchanges are set as $J_{n}^{(2)} \equiv J_{n+1}^{(1)}$, and parametrized by $J_{n}^{(1)}=J_{1}\left[1+(-1)^{n} \delta_{1}\right]$, say for chain (1). To maintain purely antiferromagnetic interactions throughout the $2 L$ spins of the ladder with periodic boundary conditions, the dimerization parameters are kept bounded by $\left|\delta_{i}\right| \leqslant 1, i$ $=1,2$. The corresponding zig-zag structure is schematized in Fig. 1.

Besides its theoretical interest, ${ }^{8}$ at room temperature this triangular topology is actually realized both in $\mathrm{NH}_{4} \mathrm{CuCl}_{3}$ (Ref. 3) and $\mathrm{KCuCl}_{3}$ (Refs. 3,9) crystals. As it was referred to above, their magnetization curves exhibit quite different features thus, we are especially interested to include an homogeneous field term of the type

$$
H_{h}=-\frac{h}{2} \sum_{n}\left[S_{n}^{z(1)}+S_{n}^{z(2)}\right],
$$

so as to elucidate the combined effect of the above kinds of coupling arrays and applied magnetic fields $h$, say along the $z$ direction. Also, it is worth pointing out that anisotropic exchanges can be included straightforwardly in our bosonization procedure as well as in the numerical analysis.

In studying the ground-state regimes of different exchange parameter sets, two subcases of particular interest arise immediately. ${ }^{12,13}$ Clearly, by setting $\delta_{2}=-1$ (1) we obtain a nonfrustrated dimerized system with staggered (plain) bond alternation as indicated in Fig. 2. The analyses given in Refs. 12,13 have shown that the magnetization behavior of the former case resembles that of a single dimerized chain, as opposed to the nonstaggered or plain situation $\left(\delta_{2}=1\right)$, in which a net magnetization plateau shows up at $\langle M\rangle=1 / 2$. It will turn out that our general zig-zag ladder interpolates continuously between the above scenarios and yields rather robust plateaus at $\langle M\rangle=0$ and $1 / 2$. However, the fine tuning of exchanges can suppress these massive regimes and furthermore, for particular subsets of the param-

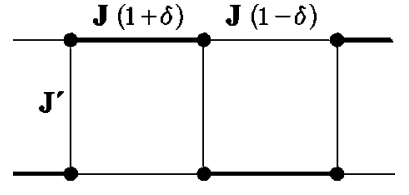

a)

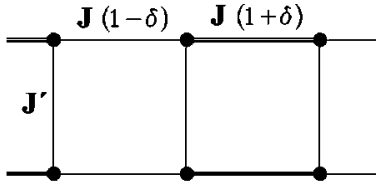

b)
FIG. 2. Representation of nonfrustrated ladders with dimerized chain bonds $J_{1}\left(1 \pm \delta_{1}\right) \equiv J(1 \pm \delta)$ and interchain coupling $J^{\prime}$ $=2 J_{2}$, obtained by setting (a) $\delta_{2}=-1$ (staggered dimerization) and (b) $\delta_{2}=1$ (plain dimerization). eter space, in addition two magnetization plateaus at $\langle M\rangle$ $=1 / 4$ and $3 / 4$ emerge simultaneously. This latter issue actually could shed light on the measurements reported in Ref. 3.

The layout of the paper is organized as follows. In Sec. II, we recast the low-energy excitations involved in each chain of Eq. (2) in terms of a conformal field theory of a free bosonic field compactified at a magnetization-dependent radius. The dimerization along chains $\left(\delta_{1}\right)$ and zig-zag interactions $\left(\delta_{2}\right)$ render the bosonization approach particularly suitable to examine, respectively, weak $\left(J_{2} / J_{1},\left|\delta_{1}\right| \ll 1\right)$ and strong $\left(J_{1} / J_{2},\left|\delta_{2}\right| \ll 1\right)$ coupling regimes. They are treated in turn in Sec II A and II B. Section III complements the magnetization behavior conjectured by the analytic approach in a variety of nonperturbative scenarios. An exact numerical treatment of magnetization contours for finite systems up to $2 L=24$ spins is given using a recursion-type Lanczos algorithm $^{14}$ applied on each magnetization subspace. Standard extrapolation procedures ${ }^{15}$ to the thermodynamic limit then enable an independent test of the results obtained via bosonization techniques. We end the paper with Sec. IV which contains our conclusions, along with some remarks on the pros and cons of the present work.

\section{ABELIAN BOSONIZATION}

Following a recent analysis discussed as in Refs. 4-8, we will apply the by now standard method of Abelian bosonization at Hamiltonian (2). In this formalism an antiferromagnetic homogeneous chain is described by a compactified free bosonic field $\phi^{(a)}$ whose dynamics is governed by

$$
H^{(a)}=\frac{1}{2} \int d x\left(v K\left(\partial_{x} \widetilde{\phi}^{(a)}\right)^{2}+\frac{v}{K}\left(\partial_{x} \phi^{(a)}\right)^{2}\right) .
$$

The dual field $\widetilde{\phi}^{(a)}$ is defined as usual $\Pi=\partial_{x} \widetilde{\phi}^{(a)} . v$ is the Fermi velocity and the Luttinger constant $K$, which is a function of the magnetization $\left\langle M^{(a)}\right\rangle$ and an eventual $X X Z$ anisotropy $\Delta$, governs the conformal dimensions of the bosonic vertex operators and can be obtained exactly from the Bethe ansatz solution of the $X X Z$ chain (see, e.g., (Ref. 5) for a detailed summary). It is related to the compactification radius $R$ of ${ }^{5}$ by $K^{-1}=2 \pi R^{2}$.

In terms of these fields, the spin operators read

$$
\begin{aligned}
& S_{x}^{z,(a)} \sim \frac{1}{\sqrt{2 \pi}} \partial_{x} \phi^{(a)}+a: \cos \left(2 k_{F}^{i} x+\sqrt{2 \pi} \phi^{(a)}\right):+\frac{\left\langle M^{(a)}\right\rangle}{2}, \\
& S_{x}^{ \pm,(a)} \sim(-1)^{x}: e^{ \pm i \sqrt{2 \pi} \widetilde{\phi}^{(a)}} \\
& \times\left(b \cos \left(2 k_{F}^{(a)} x+\sqrt{2 \pi} \phi^{(a)}\right)+c\right),
\end{aligned}
$$

where the colons denote normal ordering with respect to the ground state with magnetization $\left\langle M^{(a)}\right\rangle$. The Fermi momentum $k_{F}^{(a)}$ is related to the magnetization of the $a$ th chain as $k_{F}^{(a)}=\left(1-\left\langle M^{(a)}\right\rangle\right) \pi / 2$. The effect of an $X X Z$ anisotropy $\Delta$ and/or the external magnetic field is then to modify the scaling dimensions of the physical fields through $K$ $=K\left(\left\langle M^{(a)}\right\rangle, \Delta\right)$. The magnetization also modifies the com- 
mensurability properties of the spin operators through $k_{F}$, as can be seen from Eqs. (5),(6). The nonuniversal constants $a$, $b$, and $c$ can be in general computed numerically (see, e.g., Ref. 16, for the case of the zero magnetic field) and in particular the constant $c$ has been obtained exactly in Ref. 17.

Notice that the inclusion of an $X X Z$ anisotropy in our study is not only motivated to generalize the analysis but primarily by the fact that, for nonzero magnetization, the $\mathrm{SU}(2)$ symmetry is broken from the beginning. As we shall see in Sec. II A, the particular $\mathrm{SU}(2)$ symmetric case, $(\Delta$ $=1,\langle M\rangle=0$ ), has to be analyzed differently since our analysis breaks explicitly this symmetry. We address the reader to
Ref. 18 where the full symmetric was analyzed using a formulation in terms of Majorana fermions.

\section{A. Weak interchain coupling regime}

Here we take $\alpha \equiv J_{2} / J_{1} \ll 1$ and $\delta_{1} \ll 1$. In this regime $\left|\delta_{2}\right| \ll 1$ corresponds to a weakly coupled two-leg zig-zag ladder made up of dimerized chains, whereas for $\left|\delta_{2}\right| \rightarrow 1$ the system approaches a staggered $\left(\delta_{2}=-1\right)$ or plain $\left(\delta_{2}=1\right)$ ladder dimerized as in Figs. 2(a) and 2(b), respectively.

Using Eqs. (5),(6), the low-energy Hamiltonian can be written in this regime as

$$
\begin{aligned}
H_{\text {int }}^{(\alpha \ll 1)} \approx & \lambda_{1} \sum_{x} \partial_{x} \phi^{(1)} \partial_{x} \phi^{(2)}+\lambda_{2} \sum_{x} \cos \left[4 k_{F} x+\sqrt{2 \pi}\left(\phi^{(1)}+\phi^{(2)}\right)\right]+\lambda_{3} \sum_{x} \cos \left[\sqrt{2 \pi}\left(\phi^{(1)}-\phi^{(2)}\right)\right] \\
& +\lambda_{4} \sum_{x} \cos \left[\sqrt{2 \pi}\left(\widetilde{\phi}^{(1)}-\widetilde{\phi}^{(2)}\right)\right]+\lambda_{5} \sum_{x,(a)}(-1)^{x+a} \cos \left[2 k_{F}(x+1 / 2)+\sqrt{2 \pi} \phi^{(a)}(x)\right] \\
& +\lambda_{6} \sum_{(a)=1}^{2} \sum_{x} \cos \left[2 k_{F} x+\sqrt{2 \pi} \phi^{(a)}(x)\right],
\end{aligned}
$$

where

$$
\lambda_{j} / \alpha \propto\left\{\begin{array}{l}
\Delta_{2}, \quad j=1, \\
\Delta_{2}\left[\left(1-\delta_{2}\right)+\left(1+\delta_{2}\right) \cos \left(2 k_{F}\right)\right], \quad j=2,3, \\
\delta_{2}, \quad j=4, \\
\delta_{1}, \quad j=5, \\
\langle M\rangle, \quad j=6 .
\end{array}\right.
$$

In the last two expressions the proportionality factors are nonuniversal functions of the $X X Z$ anisotropy $\Delta_{2}$, the magnetization $\langle M\rangle$ and $\delta_{2}$.

In the above equation we have suppressed marginal terms, due to the presence of more relevant interactions. Also in the case of nondimerized zig-zag interactions, there are parity breaking terms, discovered in Ref. 18, which should be analyzed differently since they have nonzero conformal spin. However, by explicitly including these latter terms in the renormalization-group computations, it can be shown that they do not change the conclusions presented here. The SU(2) symmetric case $(\Delta=1,\langle M\rangle=0)$ has been studied in Ref. 18 for the case of zero zig-zag dimerization.

Let us analyze the structure of the magnetization curves predicted by this effective Hamiltonian. The relative field $\phi_{-}=\phi_{1}-\phi_{2}$ is always massive due to the fact that the relevant perturbation terms $\lambda_{3,4}$ are always commensurate. The analysis for the diagonal field $\phi_{+}=\phi_{1}+\phi_{2}$ is more subtle so we will consider separately different values for the magnetization. The possible values of the magnetization at which plateaus can appear are given by the general expression (1) ${ }^{4,5}$ However, due to the presence of frustration in certain region of the parameter space, possibly Eq. (1) should include an extra factor 2 to account for a possible enhancement in the periodicity of the ground state. This restricts the set of possible magnetization plateaus to the values $\langle M\rangle$ $=0,1 / 4,1 / 2,3 / 4$ (apart from saturation).

For $\langle M\rangle=0$, (i.e., $k_{F}=\pi / 2$ ), the perturbation $\lambda_{2}$ becomes commensurate and hence opens a gap for the diagonal field $\phi_{+}$. One could, however, argue that taking into account other (radiative) corrections (coming mainly from $\lambda_{5}$ and $\lambda_{6}$ ) one could close the $\phi_{+}$gap, simply by making the amplitude of this perturbation term to vanish. This naive analysis has been confirmed by numerical computations, ${ }^{13}$ showing that there exists a whole curve in the parameter space where this indeed happens. In particular, for $\delta_{2}=-1$ the critical line turns out to be

$$
\frac{J_{2}}{J_{1}} \propto \delta_{1}^{2} .
$$

This phenomenon was originally suggested in Ref. 12 using nonlinear $\sigma$ model techniques and has been also studied numerically in Refs. 20,21.

For $\langle M\rangle=1 / 2$, (i.e., $k_{F}=\pi / 4$ ), the plateau can open due to the radiatively generated (relevant) terms, (coming from $\lambda_{5}$ and $\lambda_{6}$ ), which are of the form

$$
\alpha^{2} \delta_{1}\langle M\rangle f\left(\delta_{1}, \delta_{2}, \Delta_{2}\right)(-1)^{x} \cos \left(4 k_{F} x+\sqrt{2 \pi} \phi_{+}\right),
$$

where $f\left(\delta_{1}, \delta_{2}, \Delta_{2}\right)$ vanishes for the case of $\delta_{2}=-1$, as already pointed out in Ref. 13. As it will be shown in Sec. III, possibly there is another point at which the $\langle M\rangle=1 / 2$ plateau closes. The existence of such effects can be predicted using the bosonization formalism, but the precise location of this point, however, cannot be obtained due to the presence of nonuniversal constants in Eqs. (5) and (6).

The case of $\langle M\rangle=1 / 4,3 / 4$, (i.e., $k_{F}=3 \pi / 8, \pi / 8$ ), is less clear since the commensurate operators that can be generated to open this plateau are irrelevant. One possible candidate is 


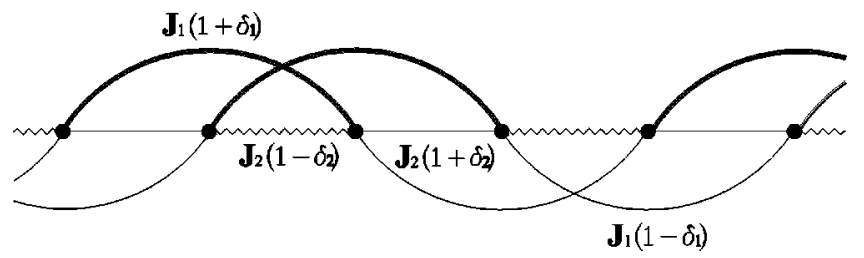

FIG. 3. Chain representation of dimerized zig-zag ladder.

the operator $(-1)^{x} \cos \left(8 k_{F} x+2 \sqrt{2 \pi} \phi_{+}\right)$which is irrelevant. One can speculate whether this operator can become relevant in some region of the parameter space by taking into account the $\lambda_{1}$ perturbation which changes the compactification radius of the $\phi_{+}$field, and hence the dimensions of the perturbation terms. However, in first approximation the above mentioned operator would become relevant for values of the couplings far from the region where our approach can be considered valid.

\section{B. Strong interchain coupling regime}

Let us consider now the opposite regime, i.e., $\alpha \gg 1$. In this regime it is convenient to rewrite the two-leg zig-zag ladder Hamiltonian as that of a single chain with alternating intrachain coupling $J_{2}\left(1 \pm \delta_{2}\right)$ and next-nearest-neighbor $(\mathrm{NNN})$ interactions $J_{1}\left(1 \pm \delta_{1}\right)$ but alternating every two sites. Namely, NNN spins at $(4 n, 4 n+2)$ and $(4 n+1,4 n$ $+3)$ pair locations are coupled by $J_{1}\left(1-\delta_{1}\right)$, whereas spins at $(4 n-2,4 n)$ and $(4 n-1,4 n+1)$ interact through $J_{1}(1$ $\left.+\delta_{1}\right)$. This is illustrated in Fig. 3.

Using the same approach as described above, in the present case we get an effective Hamiltonian for a single bosonic field perturbed by the following terms:

$$
\begin{aligned}
H_{\mathrm{int}}^{(\alpha \gg 1) \approx} & \sum_{x} \gamma_{1}(x)\left(\partial_{x} \phi\right)^{2}+\sum_{x} \gamma_{2}(x) \cos \left[2 k_{F} x\right. \\
& +\sqrt{2 \pi} \phi(x)]+\gamma_{3} \sum_{x}(-1)^{x} \cos \left[2 k_{F}(x+1 / 2)\right. \\
& +\sqrt{2 \pi} \phi(x)]+\sum_{x} \gamma_{4}(x) \cos \left[4 k_{F} x+2 \sqrt{2 \pi} \phi(x)\right] .
\end{aligned}
$$

Here $\gamma_{1,2,4}(x)$ are proportional to $\delta_{1}$ and $\gamma_{3}$ to $\delta_{2}$. The terms proportional to $\delta_{1}$ have an extra modulation due to the alternation of the NNN couplings referred to above, which hereafter we call "two-by-two" modulation.

For $\delta_{1}=0$ and $\langle M\rangle=0$ only the last term is commensurate and it has dimension 2 in the isotropic case $(\Delta=1)$. This term is the one responsible for the opening of the gap at zero magnetization in the NNN antiferromagnetic Heisenberg chain, where, as it is well known, the gap opens at a critical value of the NNN coupling through a KosterlitzThouless (KT) transition. Its effect would have been the same in the present case, but due to the presence of the dimerization $\delta_{1}$ along the chain, a gap will always be present. This will be corroborated in Sec. III.

For $\langle M\rangle=1 / 2$, the perturbation $\gamma_{2}$ survives the continuum limit due to the extra "two-by-two" alternating factor, and will hence be responsible for the plateau at this value of the magnetization.
The case of $\langle M\rangle=1 / 4,3 / 4$ is again more subtle in this limit, and it can be seen that the operator that could be responsible for the appearance of these plateaus is generated from a combined effect of the chain dimerization $\left[(-1)^{x} \delta_{2}\right]$ and the two-by-two alternating part of the NNN exchange $\left( \pm \delta_{1} J_{1}\right)$. The operator generated through this mechanism is proportional to $\cos (2 \sqrt{2 \pi} \phi(x))$, which is irrelevant, and could become relevant at certain critical value of the coupling $\left(\propto J_{1} \delta_{1} \delta_{2}\right)$. Again, this KT point is not reachable within our perturbative approach.

\section{NUMERICAL ANALYSIS OF FINITE SYSTEMS}

To enable an independent check of the magnetization scenario obtained within the bosonization approach, we now turn to a numerical finite-size analysis of the original ladder Hamiltonian (2). A number of numerical studies of triangular ladders (or equivalently, of Heisenberg chain with NN exchanges), have been reported already (Refs. 8,22 and references therein). However, the effect of dimerization along both interchain and intrachain couplings, which is crucial for the appearance of nontrivial magnetization plateaus, yet requires further numerical efforts.

We focus attention on the ground-state energy obtained from an exact diagonalization of finite systems via a recursion type Lanczos algorithm ${ }^{14}$ applied on each magnetization subspace with $S^{z}=\{0,1, \ldots, L\}$. Since the magnetic field considered in Eq. (3) is coupled to the conservation of $S^{z} / L=\langle M\rangle$, we can readily relate the energy per spin $e_{h}$ at finite fields to those at $h=0$ just by taking $e_{h} \equiv e_{0}-h\langle M\rangle$. Thus, all results addressed below were obtained from computations with $h=0$. Also, to avoid unwanted effects introduced by both the ladder topology and periodic boundary conditions, even multiples of ladder lengths up to $L=12$ spins were taken throughout.

The huge dimensionality of the spaces involved, growing as $\left(\begin{array}{l}2 L \\ L\end{array}\right)$, constrained us to use in the heaviest situations, i.e., $S^{z}=0,1$, at most seven Lanczos vectors per tridiagonalization cycle. ${ }^{19}$ Nevertheless, the numerical accuracy was kept bounded by $10^{-7} \mathrm{~h} / \mathrm{J}_{1}$ employing typically up to 40 cycles of recursion. On the other hand, the rather small number of Lanczos vectors used in the computations allowed for an efficient management of a complete, vector by vector reorthogonalization. As is known, ${ }^{14}$ this latter procedure becomes crucial to avoid the emergence of spurious eigenvalues caused by machine rounding errors which tend to build up exponentially with the number of iterations, no matter what precision is used. In what follows we limit our analysis to isotropic coupling exchanges, though preliminary calculations including anisotropy in the field direction yield qualitatively similar results.

We begin by examining the validity of Eq. (1) and test the "fine tuning" effects conjectured within the bosonization analysis. Upon setting $\delta_{1}=0.7, \delta_{2}=0.475$, and $J_{2} / J_{1}=1$, the application of the Lanczos procedure to the triangular ladder Hamiltonian (2) yields a quite unusual behavior. Specifically, in addition to the typical $\langle M\rangle=0$ and $1 / 2$ plateaus, two massive phases appear simultaneously at $\langle M\rangle=1 / 4$ and $3 / 4$ as is shown in Fig. 4. Rather unexpectedly, at this point of the parameter space the spin ladder seems to decouple into a quasi-four-level system (see the region near the vertical 


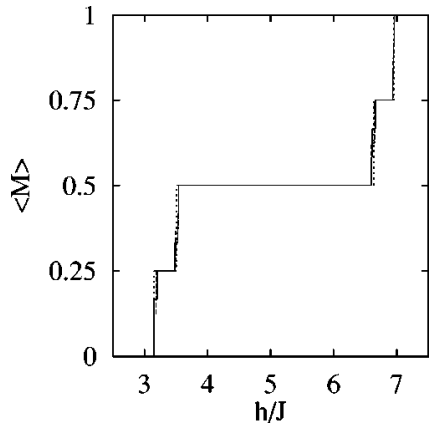

FIG. 4. Magnetization curves of dimerized zig-zag ladders for $\delta_{1}=0.7, \quad \delta_{2}=0.475$, and $J=J_{1}=J_{2}$. Solid, dashed, and short dashed lines denote, respectively, results for $L=12,8$, and 4 .

lines of Fig. 5). In principle, this renders size effects less pronounced, possibly due to some enhanced or hidden symmetry whose origin is yet difficult to elucidate. Nevertheless, our results provide a strong numerical evidence indicating that zig-zag structures can yield nontrivial magnetization plateaus such as those observed in $\mathrm{NH}_{4} \mathrm{CuCl}_{3}$ crystals. ${ }^{3}$

Interesting as it is, we are actually motivated to obtain the whole magnetization curve of this latter compound. Therefore, we turn to the scanning of the exchange parameter space for different regimes. Figures 5(a), 5(b), and 5(c) display, respectively, typical magnetization contours or "phase diagrams" for weak $\left(J_{2} / J_{1}=0.5, \delta_{1}=0.9\right)$, intermediate $\left(J_{2} / J_{1}=1, \delta_{1}=0.7\right)$, and strong $\left(J_{2} / J_{1}=5, \delta_{1}=0.5\right)$, coupling regimes. This is a compact form of representing conventional magnetization curves for a wide range of interchain couplings. Here each line is associated to successive values of $\langle M\rangle$ which increase monotonically with the applied field $h$. For example, the magnetization plateaus of Fig. 4 are contained completely within the vertical line of Fig. 5(b). In general, we found that the $\langle M\rangle=0$ and $1 / 2$ plateaus remain robust in a variety of scenarios, though their widths can be eventually "fine tuned" to yield massless gaps.

In studying the mass gap extrapolation towards their thermodynamic limits (i.e., the energy gap to create an excitation of total spin $S=1$ as $L \rightarrow \infty$ ), we fitted the whole set of finite-size results for $4 \leqslant L \leqslant 12$ ( $L$ even), using a variety of standard procedures. These range from linear to logarithmic and van den Broeck-Schwartz-type methodologies of convergence, ${ }^{15}$ which basically yield analogous results with at least two significant digits. We draw the reader's attention to Figs. 6(a) and 6(b), 6(c) and 6(d), and 6(f) and 6(g) in which we display, respectively, gap extrapolations around $\langle M\rangle=1 / 2$ and 0 corresponding to the ground-state regimes exhibited in Figs. 5(a)-5(c). Similar gap extrapolations for $\langle M\rangle=1 / 4$ and 3/4 beyond the "symmetry" or finite-size collapse region denoted by the vertical lines of Figs. 5(a) and 5(b), would be unreliable given the scarcity of available data. In our case, this is translated in the availability of matching sizes, namely $L=4,8,12$, already constrained by the studied values of $\langle M\rangle$. Thus, it remains unclear whether or not empty wide "bands" or plateau regions for $\langle M\rangle=1 / 4$ and $3 / 4$ could be actually present in Figs. 5(a) $-5(\mathrm{c})$.

To complement the analysis of finite (vanishing) $\langle M\rangle$ $=1 / 2$ gaps for $\delta_{2}=1(-1)$ given in Sec II A, we see that their widths remain stable upon setting $\delta_{2}<1,\left(\delta_{2}>-1\right)$.
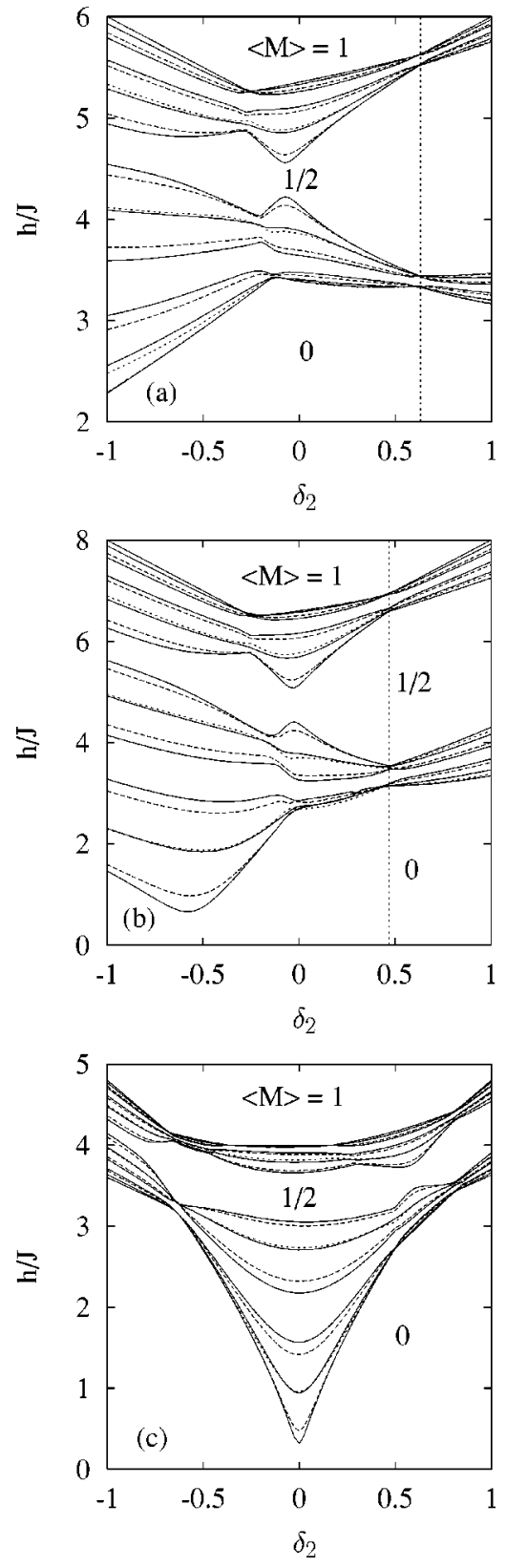

FIG. 5. Critical fields of dimerized zig-zag ladders for (a) $J_{2} / J_{1}=0.5$ with $\delta_{1}=0.9$, (b) $J_{2} / J_{1}=1$ with $\delta_{1}=0.7$, and, (c) $J_{2} / J_{1}=5$ with $\delta_{1}=0.5$. Solid, dashed, and short dashed lines denote, respectively, the results for $L=12,8$, and 4 , whereas $J$ $=\max \left\{J_{1}, J_{2}\right\}$. Vertical lines in (a) and (b) indicate regions where $1 / 4$ and $3 / 4$ plateaus emerge at a time.

Moreover, the wide minima of Figs. 6(a), 6(c), and 6(e) suggest an infinitely continuous ground-state transition at $\delta_{2}=$ -1 , which is in line with the KT singularity conjectured in Sec. II B. In fact, our data strongly support this picture for a variety of coupling regimes, at least within the region $\delta_{2}$ $\in(-0.9,-0.25)$ where finite-size effects are less pronounced. This can be observed from the semilog representation of the data displayed in Fig. 7. Thus finally, the triangular ladder turns out to interpolate smoothly between the staggered $\left(\delta_{2}=-1\right)$ and plain $\left(\delta_{2}=1\right)$ dimerization arrays of the square ladders studied in Ref. 13. 

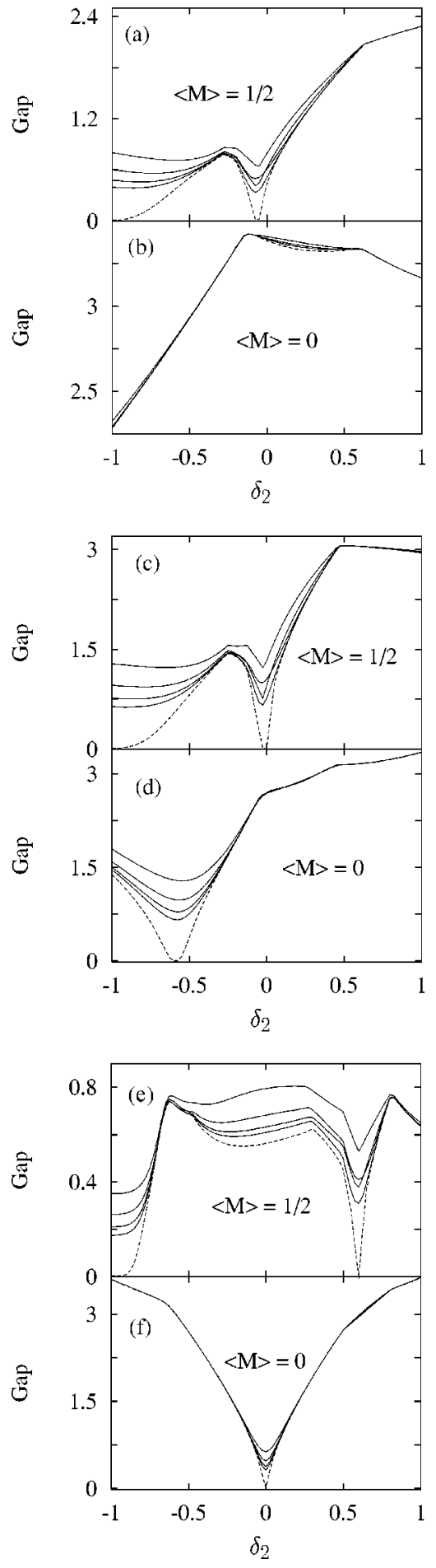

FIG. 6. Width of magnetization plateaus for $\langle M\rangle=1 / 2$ in (a), (c), (e) and $\langle M\rangle=0$ in (b), (d), (f). Data of first, second, and third panel refer, respectively, to $J_{2} / J_{1}=0.5$ with $\delta_{1}=0.9, J_{2} / J_{1}=1$ with $\delta_{1}=0.7$, and $J_{2} / J_{1}=5$ with $\delta_{1}=0.5$. Solid lines in descending order indicate, respectively, results for $L=6,8,10$, and 12, whereas lowermost dashed curves [slightly visible in (b) and (f)] denote gap extrapolation to the thermodynamic limit.

\section{CONCLUSIONS}

In this work we have studied the magnetization phase diagram of a two-leg zig-zag ladder with dimerization both along the legs and the zig-zag coupling, by means of Abelian bosonization methods complemented by Lanczos diagonal-

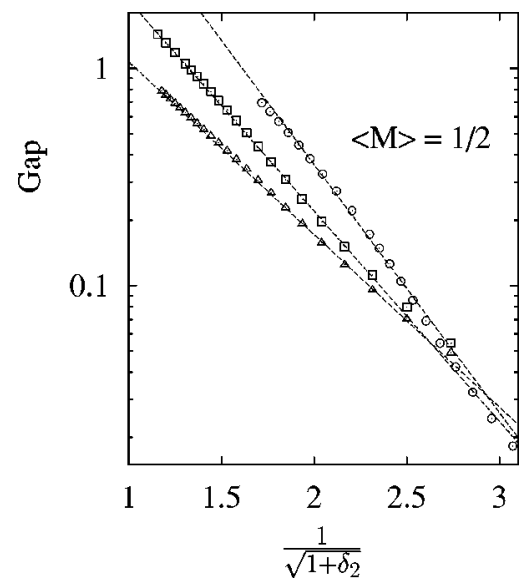

FIG. 7. Gap width extrapolations for $\langle M\rangle=1 / 2$ suggesting a KT singularity $\propto e^{-1 / \sqrt{1+\delta_{2}}}$ on approaching $\delta_{2}=-1$. Triangles, squares, and circles denote, respectively, weak, intermediate, and strongcoupling regimes displayed in turn in Figs. 6(a), 6(c), and 6(e). Straight lines are guides to the eye.

ization of finite clusters up to 24 spins. From the bosonization analysis we conclude that the $\langle M\rangle=0$ plateau is robust and it is present in the full parameter space, except on a certain zero-measure set. We have confirmed numerically these expectations and obtained the above massless excitations as displayed in the lowermost panels of Figs. 6(d) and 6(f).

Spin excitations around $\langle M\rangle=1 / 2$, also turn out to be massive and robust as they show up in the whole parameter space, except in the limit $\delta_{2} \rightarrow-1$ and for a certain curve $J_{2} / J_{1}=f\left(\delta_{1}, \delta_{2}\right)$. This latter feature is observed in Figs. 6(a) and $6(\mathrm{c})$ for weak and intermediate coupling regimes near $\delta_{2}=0$ and around $\delta_{2} \sim 0.5$ for strong-coupling regions as shown in Fig. 6(e).

Regarding the issue of $\langle M\rangle=1 / 4,3 / 4$ plateaus, they are observed only within a fine-tuned region, possibly bearing an enhanced symmetry of the Hamiltonian (see, e.g., Figs. 4 and 5). Their appearance is hard to predict using Abelian bosonization techniques.

Finally, though there are intermediate values of $\delta_{2}$ capable of closing gaps around both $\langle M\rangle=0$ and $1 / 2$, after scanning a representative set of the parameter space, we could not find any evidence of common closing points so as to explain the suppression of these plateaus at a time in $\mathrm{NH}_{4} \mathrm{CuCl}_{3}$ crystals. In principle, this would rule out the ladder Hamiltonian (2) as a suitable model to account completely for the experiments reported in Ref. 3. Nonetheless, we trust that its success to describe the simultaneous emergence of rather unusual plateaus at $\langle M\rangle=1 / 4$ and $3 / 4$ (at least near the data collapse region of Fig. 5), will make our system worth to consider as an antecedent for future studies in that direction.

\section{ACKNOWLEDGMENTS}

We appreciate fruitful discussions with A. Honecker and P. Pujol. The authors acknowledge financial support of CONICET and Fundación Antorchas. D.C.C. acknowledges financial support from ANPCyT (under Grant No. 03-0000002249). 
${ }^{1}$ The status of the field has been reviewed by T.M. Rice, Z. Phys. B: Condens. Matter 103, 165 (1997); E. Dagotto and T.M. Rice, Science 271, 618 (1996).

${ }^{2}$ Z. Hiroi et al., J. Solid State Chem. 95, 230 (1991).

${ }^{3}$ H. Tanaka et al., Physica B 246, 230 (1998); W. Shiramura et al., J. Phys. Soc. Jpn. 67, 1548 (1998).

${ }^{4}$ M. Oshikawa, M. Yamanaka, and I. Affleck, Phys. Rev. Lett. 78, 1984 (1997).

${ }^{5}$ D.C. Cabra, A. Honecker, and P. Pujol, Phys. Rev. Lett. 79, 5126 (1997); Phys. Rev. B 58, 6241 (1998), and references therein.

${ }^{6}$ D.C. Cabra and M.D. Grynberg, Phys. Rev. B 59, 119 (1999).

${ }^{7}$ K. Totsuka, Phys. Lett. A 228, 103 (1997); Phys. Rev. B 57, 3454 (1998).

${ }^{8}$ D.C. Cabra, A. Honecker, and P. Pujol, Eur. Phys. J. B 13, 55 (2000)

${ }^{9}$ H. Tanaka et al., Physica B 237, 120 (1997); K. Takatsu et al., J. Magn. Magn. Mater. 177-181, 697 (1998); T. Kato et al., J. Phys. Soc. Jpn. 67, 752 (1998).

${ }^{10}$ Notice, however, that recent experimental studies, i.e., N. Cavadini et al., Eur. Phys. J. B 7, 519 (1999), indicate relevant 3D effects in this compound.

${ }^{11}$ R. Coldea et al., Phys. Rev. Lett. 79, 151 (1997); J. Phys.: Condens. Matter 40, 7473 (1996).
${ }^{12}$ M.A. Martín-Delgado, R. Shankar, and G. Sierra, Phys. Rev. Lett. 77, 3443 (1996).

${ }^{13}$ D.C. Cabra and M.D. Grynberg, Phys. Rev. Lett. 82, 1768 (1999).

${ }^{14}$ For an account of this time honored technique see, for example, G.H. Golub and C.F. Van Loan, Matrix Computations, 3rd ed. (Johns Hopkins University Press, Baltimore, 1996).

${ }^{15}$ For a review, consult A.J. Guttmann, in Phase Transitions and Critical Phenomena, edited by C. Domb and J. Lebowitz (Academic, New York, 1990), Vol. 13; See, also, C.J. Hamer and M.N. Barber, J. Phys. A 14, 2009 (1981).

${ }^{16}$ T. Hikihara and A. Furusaki, Phys. Rev. B 58, R583 (1998).

${ }^{17}$ S. Lukyanov and A. Zamolodchikov, Nucl. Phys. B 493, 571 (1997).

${ }^{18}$ A.A. Nersesyan, A.O. Gogolin, and F.H.L. Essler, Phys. Rev. Lett. 81, 910 (1998).

${ }^{19}$ Certainly, a minimum of two Lanczos vectors will do, though at the expense of a significant amount of CPU time.

${ }^{20}$ M.A. Martín-Delgado, J. Dukelsky, and G. Sierra, Phys. Lett. A 250, 430 (1998).

${ }^{21}$ V.N. Kotov, J. Oitmaa, and Z. Weihong, Phys. Rev. B 59, 11377 (1999).

${ }^{22}$ C. Gerhardt, K.H. Mütter, and H. Kröger, Phys. Rev. B 57, 11 504 (1998). 